\title{
SMART diabetes: the way to go (Safe and Multifactorial Approach to reduce the Risk for Therapy in diabetes)
}

\author{
Ernesto Maddaloni • Paolo Pozzilli
}

Received: 11 November 2013/Accepted: 13 November 2013/Published online: 1 January 2014

(C) Springer Science+Business Media New York 2013

\begin{abstract}
Prevalence of type 2 diabetes is increasing worldwide. and management of diabetes is becoming increasingly complex because of the complexity of pathophysiology and the wide therapeutic options. The management of this complexity is difficult because of the different cardiometabolic features of patients. Thus, personalized approaches have been claimed by the most important international societies. Safety, MultifactorialApproach and reduction of Risk are the three ingredients to get the best Therapy for Diabetes, abbreviated in SMART, as every physician involved in the management of type 2 diabetes should be.
\end{abstract}

Keywords Diabetes - Safety - Personalized therapy · Multifactorial Approach

Type 2 diabetes (T2D) is not just dysglycaemia. In diabetic patients, a complex interplay of pathological mechanisms operates involving multiple organs. Management of this complexity is difficult because such interplay differs in each patient. The most significant trials in T2D produced elusive results regarding the best glucose-control strategies [1]. Results of UKPDS and PROACTIVE studies initially addressed therapeutic strategies towards an intensive glycaemic control ('the lower, the better'), but between 2008 and

E. Maddaloni · P. Pozzilli $(\bowtie)$

Department of Endocrinology \& Diabetes, Università Campus

Bio-Medico di Roma, Via Alvaro del Portillo 21, 00128 Rome,

Italy

e-mail: p.pozzilli@unicampus.it

P. Pozzilli

Centre for Diabetes, Barts and the London School of Medicine,

Queen Mary, University of London, London, UK
2009, the publication of data from ACCORD, ADVANCE and VADT trials radically changed the picture [1]. Eventually, the results are discordant likely because of the different features of subjects enrolled, but overall they raise insecurity in choosing the best therapeutic approach in terms of therapeutic targets and drugs to be used. Thus, diverse approaches to personalize therapy have been proposed [2], and a recent joint ADA/EASD position statement suggest to personalizing the glycaemic target on the basis of patients' features [3]. However, few data are available about the feasibility, and the effectiveness of personalized therapies for T2D [4] and new trials testing patient-tailored approaches are claimed to drive clinicians in the pitfalls of the available Evidence-Based Medicine (EBM). In the face of existing limitations, we propose some evergreen landmarks to correctly tailor therapies in subjects with type 2 diabetes.

Safety is the first factor a physician should contemplate; secondly, given the complexity of alterations worsening morbidity and mortality in T2D, a Multifactorial Approach is needed; finally, the actual cardio-metabolic Risk of each patient should always be stated to set up a reliable objective that should be gained with Therapy.

So Safety, Multifactorial Approach and reduction of Risk are the three ingredients to get the best Therapy for Diabetes, abbreviated in SMART as every physician involved in the management of type 2 diabetes should be to ameliorate their quality of care.

\section{Safety}

Not to cause harm and injury is a Hippocratic axiom at the basis of the current non-maleficence principle, which is as timely as ever in diabetology. Several therapeutic options are available for diabetes; the challenge for diabetologists is to choose the best option for each patient with concerns 
to potential adverse effects and uncertainties regarding the benefits of intensive glucose control. While an overall vision of available data shows that an intensive glycaemic control could damage patients with overt cardiovascular disease, suggesting that HbAlc should not be very low in patients with high cardiovascular risk [3], several concerns are rising about the safety of such antidiabetic drugs: the newest (but not the only) about the risk of pancreatitis and heart failure associated with incretin-based therapy [5]. Researches aimed towards finding out what can increase the risk of adverse events will help physicians to prescribe the right drug to the right patient. One of the few certainties is that hypoglycaemia, associated with sudden cardiac death and overall mortality [6], should be avoided by first choosing drugs with low or null hypoglycaemic risk. Moreover, diabetic patients with chronic kidney disease (CKD) are more prone to hypoglycaemia: handling drugs in patients with CKD can be very difficult, and just repeated dose adjustments with strict monitoring may ensure safety. To date, there is a substantial unfulfilled medical need for a safe and effective hypoglycaemic therapy in patients with renal injury, claiming new studies in this respect.

\section{Multifactorial Approach (MA)}

In diabetic patients, multiple risk factors coexist. There is a universal agreement that anti-hyperglycaemic therapy should be pursued within a multifactorial risk-reduction framework. For instance, diabetic patients are likely to derive greater cardiovascular benefits from lipid lowering strategies compared with patients without diabetes. Statin and fibrate have been well documented to reduce cardiovascular death, but the crisis of certainties hit also the "sacred cow" of statin benefits: intensive-dose statin therapy has been associated with an increased diabetes risk even if cardiovascular benefits of statins seem still to exceed diabetes hazard [7]. However, MA often requires polypharmacy, which is a great problem especially in older subjects. How much will patients benefit from additional pills, considering the negative consequences associated with polypharmacy [8]? These and other issues are still unsolved and a carefully personalized physical activity program still remains the unique safe and effective MA.

\section{Risk}

Coronary heart disease (CHD) is the leading killer of diabetic patients: thus, prevention of macrovascular complications should be the driving goal of therapy. The most challenging consideration is that the risk of macrovascular complications starts to increase very early, even in the prediabetic stages, claiming for precocious management strategies. In particular, there is a need for new and precocious biomarkers of cardio-metabolic diseases to earlier identify subjects with increased cardiovascular risk.

Results of interventional trials in T2D suggest that each $1 \% \mathrm{HbA} 1 \mathrm{c}$ reduction may be associated with $15 \%$ relative risk reduction in nonfatal myocardial infarction [6] but the Origin trial surprisingly showed that maintenance of fasting glucose $<95 \mathrm{mg} / \mathrm{dl}$ has neutral effect on incidence of major cardiac events [9]. There are also few certainties about the usefulness of such cardiovascular drugs (like antithrombotic agents) to reduce CHD in T2D because diabetic subjects show a peculiar pharmacological response [10]. Explanation of these unclear results is elusive, but for sure they indicate that a careful evaluation of risks and benefits should always be performed.

\section{Therapy}

Therapy of diabetes is becoming increasingly complex because of the complexity of pathophysiology and to the wide therapeutic options. Ten pharmacological classes of oral and injectable drugs/hormones, with more than 25 molecules associable with each other in several combinations are to date available to, thereby causing comprehensively confusion for the clinician. A non-univocal, but just a SMART approach to type 2 diabetes could be the key to turn therapeutic complexity from a problem into an opportunity.

The acronym SMART (Safe and Multifactorial Approach to reduce the Risk for Therapy in diabetes) can be a useful tool to guide the daily choice of physicians in choosing the right therapy highlighting a scale of priorities in the decision making process.

Final suggestions:

A. What a SMART diabetologist can do in practice considering current limitations? Safety, Multifactorial-Approach and Risk-benefit ratio are three evergreen landmarks to correctly face EBM limits (Fig. 1).

B. What questions are still unsolved?

(1) Personalized therapies are claimed for T2D, but little evidence is available about the feasibility and the effectiveness of this approach.

(2) MA often requires multiple pills, but there are many negative consequences associated with polypharmacy: do the benefits of the adjunctive pill overcome the risk of polypharmacy?

(3) New and precocious biomarkers of cardio-metabolic diseases need to be discovered for an earlier identification of subjects at risk. 
Fig. 1 SMART

\begin{abstract}
$\mathbf{S}_{\text {afety }}$

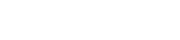

Multifacorial

A pproach

\section{"Primum non nocere"}

The challenge for diabetologist is to choose the best safe approach with concerns to potential adverse effects and benefits of intensive glucose control.

In diabetic patients relevant cardiovascular risk factors other than hyperglycaemia always coexist. There is a universal agreement that anti-hyperglycaemic therapy should be pursued within a multifactorial risk reduction framework and a carefully personalized physical activity program still remains the unique safe and effective MA
\end{abstract}

Risk

Therapy

Therapy of type 2 diabetes should be directed to reduce the risk of diabetes related complications and prevention of macrovascular complications should be the driving goal of therapy. A careful evaluation of the risk reduction that could really be achieved with antidiabetic therapy should always be performed.

Therapy of diabetes is becoming increasingly complex, due to the complexity of pathophysiology and to the wide therapeutic options. A non univocal, but just a smart approach could be the key to turn therapeutic complexity from a problem into an opportunity.
The smart diabetologist by applying these concepts in daily practice should increase the wellbeing of a diabetic patient by reducing hospital admittance, improving quality of life and saving money for care.

Conflict of interest The authors declare that they have no conflict of interest pertinent to this article.

\section{References}

1. K.K. Ray, S.R. Seshasai, S. Wijesuriya, R. Sivakumaran, S. Nethercott, D. Preiss, S. Erqou, N. Sattar, Effect of intensive control of glucose on cardiovascular outcomes and death in patients with diabetes mellitus: a meta-analysis of randomised controlled trials. Lancet 373, 1756-1772 (2009)

2. P. Pozzilli, R.D. Leslie, J. Chan, R. De Fronzo, L. Monnier, I. Raz, S. Del Prato, The A1C and ABCD of glycaemia management in type 2 diabetes: a physician's personalized approach. Diabetes/Metab. Res. Rev. 26, 239-244 (2010)

3. S.E. Inzucchi, R.M. Bergenstal, J.B. Buse, M. Diamant, E. Ferrannini, M. Nauck, A.L. Peters, A. Tsapas, R. Wender, D.R. Matthews, American Diabetes Association (ADA); European Association for the Study of Diabetes (EASD). Management of hyperglycemia in type 2 diabetes: a patient-centered approach: position statement of the American Diabetes Association (ADA) and the European Association for the Study of Diabetes (EASD). Diabetes Care 35, 1364-1379 (2012)
4. W.D. Strain, V. Lukashevich, W. Kothny, M.J. Hoellinger, M.P. Paldánius, Individualised treatment targets for elderly patients with type 2 diabetes using vildagliptin add-on or lone therapy (INTERVAL): a 24 week, randomised, double-blind, placebocontrolled study. Lancet 382, 409-416 (2013)

5. P.C. Butler, M. Elashoff, R. Elashoff, E.A. Gale, A critical analysis of the clinical use of incretin-based therapies: are the GLP-1 therapies safe? Diabetes Care 36, 2118-2125 (2013)

6. S. Zoungas, A. Patel, J. Chalmers, B.E. de Galan, Q. Li, L. Billot, M. Woodward, T. Ninomiya, B. Neal, S. MacMahon, D.E. Grobbee, A.P. Kengne, M. Marre, S. Heller, Severe hypoglycemia and risks of vascular events and death. N. Engl. J. Med. 363, 1410-1418 (2010)

7. P.M. Ridker, A. Pradhan, J.G. MacFadyen, P. Libby, R.J. Glynn, Cardiovascular benefits and diabetes risks of statin therapy in primary prevention: an analysis from the Jupiter trial. Lancet $\mathbf{3 8 0}$, 565-571 (2012)

8. R.E. Hubbard, M.S. O’Mahony, K.W. Woodhouse, Medication prescribing in frail older people. Eur. J. Clin. Pharmacol. 69, 319-326 (2013)

9. H.C. Gerstein, J. Bosch, G.R. Dagenais, R. Díaz, H. Jung, A.P. Maggioni, J. Pogue, J. Probstfield, A. Ramachandran, M.C. Riddle, L.E. Rydén, S. Yusuf, Basal insulin and cardiovascular and other outcomes in dysglycemia. N. Engl. J. Med. 367, 319-328 (2012)

10. L. Rydén, P. Grant, S.D. Anker, C. Berne, F. Consentino, N. Danchin, C. Deaton, J. Escaned, H.P. Hammes, H. Huikuri, M. Marre, N. Marx, L. Mellbin, J. Ostergren, J. Ostergren, C. Patrono, P. Seferovic, Uva, Taskinen, M. Tendera, J. Tuomilehto, P. Valensi, J.L. Zamorano, ESC Guidelines on diabetes, pre-diabetes, and cardiovascular diseases developed in collaboration with the EASD. Eur. Heart J. (2013). doi:10.1093/eurheartj/eht108 